\title{
An Adaptive Model-Based Real-time Voltage Control Process for Active Distribution Networks Using Battery Energy Storage Systems
}

\author{
Maryam Bahramipanah, Dimitri Torregrossa, Rachid Cherkaoui, Mario Paolone \\ Distributed Electrical System Laboratory (DESL) \\ Ecole Polytechnique Fédérale de Lausanne \\ Lausanne-Switzerland
}

\begin{abstract}
The paper presents a centralized real-time adaptive and model-based voltage control algorithm for Active Distribution Networks (ADNs). Differently from the available literature, the proposed algorithm merely relies on the control of Battery Energy Storage Systems (BESSs). In this respect, an experimentally model-fitted two-time constant dynamic model of BESS is used. In particular, this model is used to compute the constraints of the BESSs in terms of DC active power at each controller iteration. These constraints are subsequently used in the central controller for the solution of the optimal voltage control problem. The performances of the proposed method are compared with those obtained for the case where BESSs are modeled as ideal energy reservoirs. Such an assessment is carried out using a numerical example referring to IEEE 13 buses distribution test feeder suitably adapted to include stochastic generation and BESSs.
\end{abstract}

Index Terms-Battery Energy Storage Systems, State-of-Energy, State-of-Charge, TTC battery model, model-based voltage control.

\section{Nomenclature}

BESS Battery Energy Storage System

SoE State-of-Energy

SoC State-of-Charge

$t \quad$ Index of time

$\Delta t \quad$ Time interval

$i, j \quad$ Indexes of the grid node where the BESSs are located

$k \quad$ BESSs operation cost constant

$\varepsilon^{+} \quad$ Very small positive quantity

$\varepsilon^{-} \quad$ Very small negative quantity
$B \quad$ Set of buses where BESSs are located

$N \quad$ Set of buses in the network

$V S C_{j \rightarrow i}^{P} \quad$ Voltage Sensitivity Coefficient related to the active power variation of BESS located at bus $j$ on node $i$

$V S C_{j \rightarrow i}^{Q} \quad$ Voltage Sensitivity Coefficient related to the reactive power variation of BESS located at bus $j$ on node $i$

$E_{\text {nom }} \quad$ Rated energy of BESS

So $E_{j, t} \quad$ SoE of BESS located at bus $j$ at time $t$

SoE $E_{\text {ref }} \quad$ SoE reference value

$S o E_{j}^{\min } \quad$ Minimum $S o E$ for BESS located at bus $j$

$S o E_{j}^{\max } \quad$ Maximum $S o E$ for BESS located at bus $j$

$\bar{V}_{i, t} \quad$ Voltage of node $i$ at time $t$

$V_{\text {ref }} \quad$ Node voltages reference values

$V_{B E S S, j, t}^{d c} \quad$ DC voltage of BESS located at bus $j$ at time $t$

$V_{B E S S, j}^{d c, \min } \quad$ Minimum DC voltage of BESS located at bus $j$

$V_{B E S S, j}^{d c, \max } \quad$ Maximum DC voltage of BESS located at bus $j$

$I_{B E S S, j}^{d c, \min } \quad$ Minimum DC current of BESS located at bus $j$ (discharge)

$I_{B E S S, j}^{d c, \max } \quad$ Maximum DC current of BESS located at bus $j$ (charge)

$P_{B E S S, j, t}^{a c} \quad$ AC active power produced/absorbed by the BESS located at bus $j$ at time $t$ 
$Q_{B E S S, j, t}^{a c}$ Reactive power produced/absorbed by BESS located at bus $j$ at time $t$

$S_{c o n v, j} \quad$ Rated power of the BESS converter located at bus $j$

$P_{c o n v, j, t}^{l o s s} \quad$ Losses inside the power converter related to the BESS located at bus $j$ at time $t$.

$P_{B E S S, j, t}^{d c} \quad$ DC active power of BESS located at bus $j$ at time $t$

$P_{B E S S, j, t}^{d c, \min } \quad$ Minimum DC active power of BESS located at bus $j$ at time $t$

$P_{B E S S, j, t}^{d c, \max } \quad$ Maximum DC active power of BESS located at bus $j$ at time $t$

$P_{B E S S, j, t}^{d c, \min V} \quad$ Minimum DC power of the BESS located at bus $j$ at time $t$ accounting for just DC voltage constraints

$P_{B E S S, j, t}^{d c, \max V} \quad$ Maximum DC power of BESS located at bus $j$ at time $t$ accounting for just DC voltage constraints

$P_{B E S S, j, t}^{d c, \min I} \quad$ Minimum DC power of BESS located at bus $j$ at time $t$ accounting for just DC current constraints

$P_{B E S S, j, t}^{d c, \max } \quad$ Maximum DC power of BESS located at bus $j$ at time $t$ accounting for just DC current constraints.

\section{INTRODUCTION}

In the last decades, due to the rapid increase of connection of non dispatchable distributed renewable energy resources, the issue of real-time voltage regulation in Active Distribution Networks (ADNs) became more compelling. Integration of Distributed Generations (DGs) in distribution networks imposes different technical challenges caused by the lack of direct control over stochastic and non-stochastic DGs (e.g.,[1]). In this context, Distributed Energy Storage Systems (DESSs) have been identified as a possible nearterm solution to provide flexibility to the operation of distribution systems. Line congestion mitigation, voltage support and, eventually, spinning reserve management can be achieved through DESSs. Lithium-based Battery Energy Storage Systems (BESSs) are one of the DESS technologies matching the requirement of ADNs. Their mains advantages are the relative high value of energy density and long life duration as well as, in certain cases, limited environmental impact [2].

In the proposed work, it is assumed that the targeted distribution system does not contain any directly-controllable generators, or loads, except BESS units. Consequently, the adopted voltage control method merely relies on BESSs as controllable devices. The adopted control method involves the centralization of all the information in a single processing unit (Central Controller) [3]. The central controller gathers all the system data from sensors located in the system and computes control variables setpoints (i.e., active and reactive power) for each BESS.
In a previous work [4], the Authors of this manuscript have proposed a similar control process where DESSs have been used for the grid voltage control. In that preliminary work, the DESSs have been modeled as ideal energy reservoirs and their dynamic behavior was not taken into account. In this paper, an adaptive model-based control (MBC) relying on the accurate dynamic representation of BESSs is proposed and compared with the equivalent controller of [4]. For this purpose, an experimentally-fitted two-time constant dynamic model of Lithium-Titanate battery, is used. The locations of BESSs in the network are considered to be given and provided by the process described in [5]. Also, the cost of using BESSs for controlling ADNs has been already treated in [5] where an optimal planning accounting for the investment and operation costs of the BESSs is proposed.

The manuscript is structured as follows: Section II presents two different BESS models. Section III first describes the centralized optimal voltage control problem then, based on the BESS dynamic model presented in II, proposes a closed form process to compute the BESS constraints accounted for in the former optimal control problem. Section IV illustrates the performance evaluation of the proposed control method with reference to the IEEE 13 node test distribution feeder.

\section{BESSS DYNAMIC MODELS}

As mentioned in the introduction, if BESS are the controlled components of an ADN regulator, their modeling will inherently influence the feasibility and optimality of the regulator itself. In this respect, two general models for BESSs are presented in this section. In particular, the first model represents the typical approach adopted in the literature to represent the behavior of BESSs in ADN control problems. Whilst the second one, based on the known two-time constant model, account for the internal short-term dynamic of the targeted BESS.

\section{A. Energy Representative Model (ER)}

This type of model (e.g., [6]) does not account for the internal charge flow and diffusion processes of battery cells. Instead, it considers the cell as an ideal reservoir of charge and energy. In general, the State-of-Charge $(\mathrm{SoC})$ is used for electrochemical storage system to indicate the available amount of charge that can be extracted from the system at a given discharge rate [6]. Therefore, this quantity does not explicitly quantify the energy reservoir level of the storage system since it depends on the current value used to discharge it. Consequently, it is necessary to define a State-of-Energy $(S o E)$ indicating the energy that can be extracted from a generic storage system at any discharge rate. As a first approximation, $S o E$ is defined with a unity energy efficiency of the device using (1).

$\operatorname{SoE}(t)=\operatorname{SoE}\left(t_{0}\right)+\int_{t_{0}}^{t} \frac{P_{B E S S}^{d c}(t)}{E_{\text {nom }}} d t$ 
As a result, with this model BESSs are represented as an ideal energy reservoir. No dynamics of the BESS are considered and, therefore, its constraints are only on the $S o E$ upper and lower bounds.

It is interesting to observe that, since there is no voltage dynamic accounted in this model, charge and energy are equivalent quantities.

\section{B. Two-time constants model (TTC)}

The most common electrical model of Lithium-based electrochemical cell accounting for the internal processes associated to charge diffusion, is based on the adoption of the equivalent Thévenin-circuit (e.g., [7]). The model, shown in Figure 1 is composed of two $R C$ time constants (TTC). It is able to account for slow and fast electrochemical process ( $R_{1}, C_{1}$ for the fastest and $R_{2}, C_{2}$ for the slowest BESS charge diffusion dynamics). $R_{0}$ is the equivalent series resistance (usually also named as $E S R$ ) and $E m$ is the electromotive force of the cell which can be defined (and measured) as the voltage across the terminals without any charge connected (in the steady-state conditions). All the above parameters are not linear and are function of the cell $S o E$. This model represents one of the best tradeoff between accuracy and model complexity.

The exchanging charge between the layers inside the battery characterizes the recovery/redistribution effects during relaxation phases (e.g., [8], [9]). In order to account for the so-called charge redistribution, in [10] it has proposed to use a virtual current generator $\left(I_{v i r}\right)$ which wave shape and behavior has been suitably defined to match the long-term charge diffusion in the battery. Since in this work it is considered that the delivered/absorbed charge of the battery is continuously changed by the real-time voltage controller, the effect of the redistribution phenomena can be neglected. Therefore, the BESS TTC model adopted in this work is the one show in Fig. 1.

The system of equations governing the battery cell shown in Fig. 1 can be associated to a system of ordinary differential equations (ODE) as in (2), (3).

$$
\left\{\begin{array}{l}
\dot{V}_{1}=\frac{-1}{R_{1} C_{1}} V_{1}+\frac{1}{C_{1}} I_{d c} \\
\dot{V}_{2}=\frac{-1}{R_{2} C_{2}} V_{2}+\frac{1}{C_{2}} I_{d c} \\
V_{d c}=V_{1}+V_{2}+R_{0} I_{d c}+E m
\end{array}\right.
$$

The ODE system (2) can be represented as a system of two state variables $X=\left(V_{1}, V_{2}\right)$, where the input is $I_{d c}$ and the output is $V_{d c}$ (in the following, the state $\mathrm{X}$ is represented by the column vector $\left[V_{1} V_{2}\right]^{T}$ ).

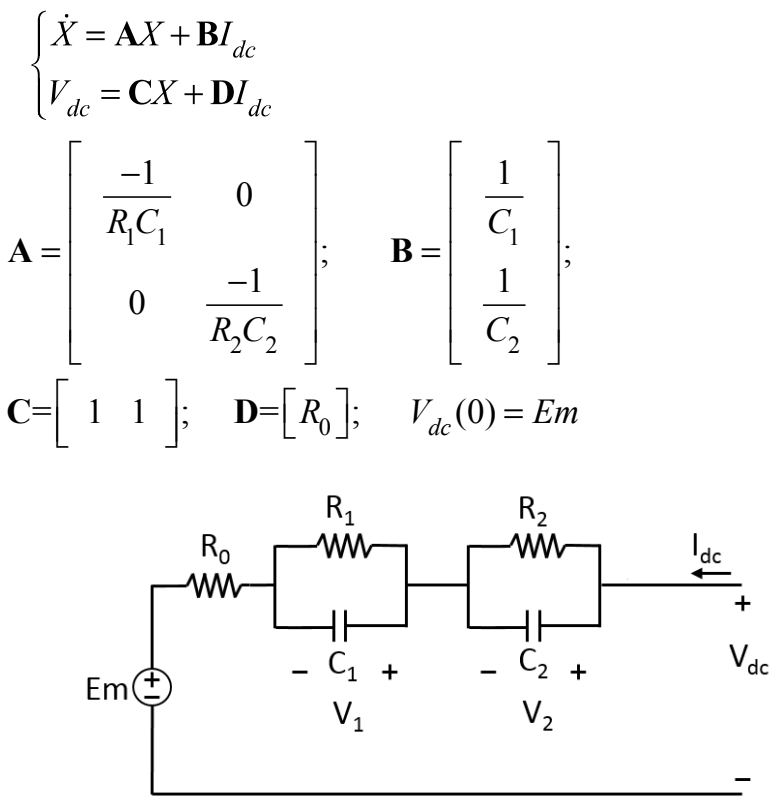

Figure. 1. The BESS TTC model.

In this paper, the energy flow is assumed to be positive during the charge and negative during the discharge. Model identification and parameter estimation are the ones described in [7], [10]. As known, all the cell parameters depend on the $S o E$ as well as on the rate of the discharge current (the socalled C-rate). For this reason, matrices $\mathbf{A}$ and $\mathbf{D}$ have been experimentally fitted for different $\mathrm{SoC}$ using the process described in [10].

\section{GRID VOLTAGE CONTROL PROCESS}

This section presents a real-time grid voltage control method accounting for the described dynamic model of the BESSs (TTC model) in section II. The location of BESSs in the system is assumed to be given (for instance, by means of the process illustrated in [5]). The control algorithm is carried out by a unique central controller located in the ADN primary substation. At each time step, the controller solves a voltage control optimal problem and it determines a feasible BESSs operating points (i.e. their active/reactive power set-points) with a refresh rate that, for instance, can be of hundreds of ms. Then, by assuming to know the $S o E$, the controller computes the operative bounds of the BESSs in terms of DC active power limits for the next time step. These bounds are included as constraints in the control optimization problem.

At the current time $t$, the central controller solves a central multi objective optimal problem formulated as (4) and sends the active/reactive $\mathrm{AC}$ power set-points to the power converter of BESSs (control variables of the optimization problem). Subsequently, the new voltage values for the grid buses are provided. The description of the control problem objective and constraints is given below. 


$$
\begin{aligned}
& \min _{P_{B E S S, j, t}^{a c}, Q_{B E S S, j, t}^{a c}} g\left(S o E_{j, t}\right)+f\left(\left|\bar{V}_{i, t}\right|\right) \\
& \text { a) } \operatorname{SoE} E_{j}^{\min } \leq S o E_{j, t} \leq \operatorname{SoE}_{j}^{\max } \\
& \text { s.t } \\
& \text { b) } P_{B E S S, j, t}^{a c}{ }^{2}+Q_{B E S S, j, t}^{a c}{ }^{2} \leq S_{c o n v, j}{ }^{2} \\
& \text { c) } P_{B E S S, j, t}^{d c, \min } \leq P_{B E S S, j, t}^{d c} \leq P_{B E S S, j, t}^{d c, \max }
\end{aligned}
$$

\section{A. Objective function:}

The objective function includes two parts: 1) the one considering the BESSs operation costs $(g)$; and 2) the one related to the grid voltage regulation $(f)$.

\section{1) BESSs operation costs:}

The operation cost of the battery is expressed with (5) as a penalty of the deviation of the $S o E$ from a reference value. Since the adopted control does not include any prediction on the load absorptions and DG injections, this choice is justified to keep the battery into an average $S o E$ condition that allows to equally absorb and provide energy with respect to the stochastic injections / absorptions.

$$
\begin{aligned}
& g\left(\operatorname{SoE}_{j, t}\right)= \\
& \sum_{j \in B, t} k\left(\operatorname{SoE}_{j, t}-\operatorname{SoE}_{r e f}\right)^{4} \cdot\left(a \cdot \operatorname{SoE}_{j, t}{ }^{2}-b \cdot \operatorname{SoE} E_{j, t}+c\right)
\end{aligned}
$$

where $a, b, c$ are constant. The cost function $g$ is shown in Fig. 2. It is seen that the cost increased significantly when the battery $S o E$ is close to the fully charged/discharged condition.

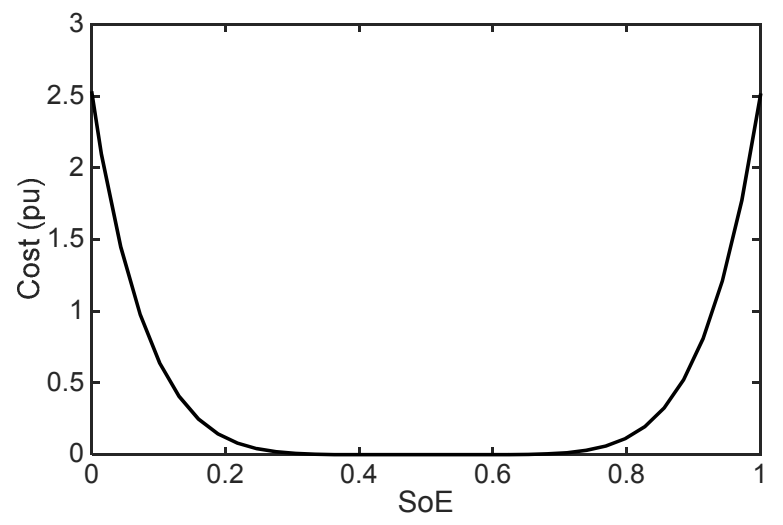

Figure. 2. The BESS cost function expressed by (5).

\section{2) Grid voltage regulation:}

In order to keep the grid nodes voltages close to the desired value, the norm of the voltage deviations are minimized for all nodes of the system at each time-step.

$$
f\left(\left|\bar{V}_{i, t}\right|\right)=\sum_{i \in N, t}\left\|\left|\bar{V}_{i, t}\right|-V_{r e f}\right\|^{2}
$$

Since the link of this objective function with the control variable is non-convex, we have adopted a linear programming approach relying on the so-called voltage sensitivity coefficients (VSC) [11]. The VSCs can be computed for both active/reactive powers of BESS located in ADNs. As a result, the voltage at bus $i$ could be expressed as its initial value $\left(\bar{V}_{i, t}^{\text {init }}\right)$ plus a variation $\left(\Delta\left|\bar{V}_{i, t}\right|\right)$ provided by the BESSs.

$\left|\bar{V}_{i, t}\right|=\left|\bar{V}_{i, t}^{i n i t}\right|+\Delta\left|\bar{V}_{i, t}\right|$

$\Delta\left|\bar{V}_{i, t}\right|$ is computed by the following (approximated) linear equation:

$\Delta\left|\bar{V}_{i, t}\right|=\sum_{j=1}^{B}\left(P_{B E S S, j, t}^{a c} \cdot V S C_{j \rightarrow i}^{P}+Q_{B E S S, j, t}^{a c} \cdot V S C_{j \rightarrow i}^{Q}\right)$

\section{B. Constraints:}

\section{1) SoE constraint (4.a)}

In general, the $S o E$ of the battery is limited in order to prevent excessive discharge rates that would damage the storage system and/or reduce its lifetime. These limits, $S o E^{\min }$ and $S o E^{\max }$, are defined by the BESS manufacturer. They are considered in (4.a).

2) PQ capability curve constraint of the power converter (4.b)

A bidirectional DC/AC converter is used to interface the storage with the grid. The power converter is assumed to operate in four quadrants. In order to model the power converter, the constraints on the $\mathrm{AC}$ active and reactive power are taken into account. The PQ capability curve of the converter is described by the inequality constraint (4.b). It is assumed that the DC/AC converter is characterized by an efficiency, $\eta$, independent of its power flow. Thus, the converter is modeled using (9) and (10).

$$
\begin{aligned}
& P_{B E S S, j, t}^{d c}=P_{B E S S, j, t}^{a c}+P_{\text {conv }, j, t}^{\text {loss }} \\
& P_{\text {conv }, j, t}^{\text {loss }}=\eta \sqrt{P_{B E S S, j, t}^{a c}+Q_{B E S S, j, t}^{a c}} \\
& \text { 3) DC active power constraint (4.c) }
\end{aligned}
$$

The TTC dynamic model of the BESSs is considered in this constraint. It should be stressed that the TTC model includes also the ER model. If we only consider the ER model for BESSs, this constraint is neglected.

The assumed update rate of the battery set-points imposed by the controller is small enough $(\Delta t=100 \mathrm{~ms})$, thus the SoE, and, consequently the battery parameters, can be considered constant between two consecutive set-point computations. This assumption is reasonable in view of the typical large size of BESS resulting from optimal planning problems (see [5] for further details).

It is worth observing that the $\mathrm{AC}$ active/reactive set-points are kept constant between two subsequent updates of the controller set-points. Therefore, by knowing the $\operatorname{SoE}$ at time $t-\Delta t$, the $S o E$ of the battery for the next time $t$ is computed using (1).

In order to account for the TTC model in this constraint, 
the operational limits of the BESS in terms of DC active power (based on DC voltage/current) are taken into account $\left(P_{B E S S, j, t,}^{d c, \min }, P_{B E S S, j, t}^{d c, \max }\right)$. These limits change dynamically with the SoE since the parameters of the TTC are function of this quantity. For this purpose, the following assumptions are considered:

- the parameters of the TTC model are known by means of the process described in [10] as a function of the $S o E$.

- The battery cells are arranged in suitable parallel and series connections to form an array of a given total energy and power capacities such that the status of one cell identifies the entire array.

At time $(t-\Delta t)$, a set of all feasible DC power set points for the next time interval $([t, t+\Delta t])$ are computed accounting for $V_{B E S S, j}^{d c, \min }, V_{B E S S, j}^{d c, \max }, I_{B E S S, j}^{d c, \min }, I_{B E S S, j}^{d c, \max }$ of the BESS located at bus $j$. These set-points are considered in order to determine the constraint (4.c).

Using (3) and (11), the $V_{B E S S, j, t}^{d c}$ is computed as a function of $P_{B E S S, j, t}^{d c}$ by (10) for the current time $t$.

$P_{B E S S, j, t}^{d c}=V_{B E S S, j, t}^{d c} \times I_{B E S S, j, t}^{d c}$

$V_{B E S S, j, t}^{d c}=f\left(P_{B E S S, j, t}^{d c}\right)=\frac{(\mathbf{C} X) \pm \sqrt{(\mathbf{C} X)^{2}+4 \mathbf{D} P_{B E S S, j, t}^{d c}}}{2}$

Considering the positive flow for charge and knowing that $V_{B E S S, j, t}^{d c}$ is always positive, the only valid solution of (12) for the charging phase is the one linked to the positive sign.

For the discharging phase (negative flow), both solutions linked to the positive and negative signs give positive $V_{B E S S, j, t}^{d c}$. As known, the battery DC voltage has continuous behavior. Thus, the continuity should stand crossing from zero (from charge to discharge or vice versa). As a result, charging with a small amount of positive DC power $\left(\varepsilon^{+}\right)$gives the same amount of DC voltage in case of discharging with small amount of negative DC power $\left(\varepsilon^{-}\right)$.

$V_{B E S S, j, t}^{d c}\left(P_{B E S S, j, t}^{d c}=\varepsilon^{+}\right)=V_{B E S S, j, t}^{d c}\left(P_{B E S S, j, t}^{d c}=\varepsilon^{-}\right)$

Since $V_{B E S S, j, t}^{d c}\left(P_{B E S S, j, t}^{d c}=\varepsilon^{+}\right)=\mathbf{C} X$, the only acceptable solution for the discharge phases is the one linked to the positive sign likewise the charging case.

To sum up, with a unique value of $P_{B E S S, j, t}^{d c}$, there is one and only one unique solution for $V_{B E S S, j, t}^{d c}$ in both charge and discharge phases using (14).

$V_{B E S S, j, t}^{d c}=f\left(P_{B E S S, j, t}^{d c}\right)=\frac{(\mathbf{C} X)+\sqrt{(\mathbf{C} X)^{2}+4 \mathbf{D} P_{B E S S, j, t}^{d c}}}{2}$
The matrices $\mathbf{C}$ and $\mathbf{D}$, as well as the system state $X$, are related to the current time step. However, for the sake of simplicity, the index of time is removed.

Note that the square root in (14) results in finding the critical DC power computed by (15).

$P_{B E S S, j, t}^{d c, \text { critical }}=\frac{-(\mathbf{C} X)^{2}}{4 \mathbf{D}}$

The $P_{B E S S, j, t}^{d c}$ should be always greater than the DC power of the critical point.

Since the function $f$ has the monotonic behavior (see proposition I), the limits of the DC power can be easily computed.

Proposition I: The function of $V_{B E S S, j, t}^{d c}=f\left(P_{B E S S, j, t}^{d c}\right)$ shown by (12) is monotonic.

\section{Proof:}

A function is monotonic if and only if it is either entirely increasing or decreasing. Therefore, for any two value of $P_{B E S S, j, t}^{d c}{ }^{(1)}$ and $P_{B E S S, j, t}^{d c}{ }^{(2)}$; if $\quad P_{B E S S, j, t}^{d c}{ }^{(2)} \geq P_{B E S S, j, t}^{d c}{ }^{(1)}$ then $V_{B E S S, j, t}^{d c}{ }^{(2)} \geq V_{B E S S, j, t}^{d c}{ }^{(1)} \quad\left(\right.$ or $\quad$ if $\quad P_{B E S S, j, t}^{d c}{ }^{(2)} \leq P_{B E S S, j, t}^{d c}{ }^{(1)}$ then $\left.V_{B E S S, j, t}^{d c}{ }^{(2)} \leq V_{B E S S, j, t}^{d c}{ }^{(1)}\right)$.

Using (14) and taking the derivative of $V_{B E S S, j, t}^{d c}$ with respect to $P_{B E S S, j, t}^{d c}$, we get:

$\frac{d V_{B E S S, j, t}^{d c}}{d P_{B E S S, j, t}^{d c}}=\frac{\mathbf{D}}{\sqrt{(\mathbf{C} X)^{2}+4 \mathbf{D} P_{B E S S, j, t}^{d c}}}$

Since the argument of the root as well as $\mathbf{D}$ are positive, $\frac{d V_{B E S S, j, t}^{d c}}{d P_{B E S S, j, t}^{d c}} \geq 0$. Thus,

$\frac{d V_{B E S S, j, t}^{d c}}{d P_{B E S S, j, t}^{d c}}=\frac{V_{B E S S, j, t}^{d c}{ }^{(2)}-V_{B E S S, j, t}^{d c}{ }^{(1)}}{P_{B E S S, j, t}^{d c}{ }^{(2)}-P_{B E S S, j, t}^{d c}{ }^{(1)}} \geq 0$

From (17), it is clear that if $P_{B E S S, j, t}^{d c}{ }^{(2)} \geq P_{B E S S, j, t}^{d c}{ }^{(1)}$, then $V_{B E S S, j, t}^{d c}{ }^{(2)} \geq V_{B E S S, j, t}^{d c}{ }^{(1)} \quad$ (or $\quad$ if $P_{B E S S, j, t}^{d c}{ }^{(2)} \leq P_{B E S S, j, t}^{d c}{ }^{(1)}$, then $\left.V_{B E S S, j, t}^{d c}{ }^{(2)} \leq V_{B E S S, j, t}^{d c}{ }^{(1)}\right)$.

In order to find the limits of the BESS DC active power, the central controller relies on the solution of the following problem: 
$\min (\max ) \quad P_{B E S S, j, t}^{d c}$

$$
\begin{aligned}
V_{B E S S, j}^{d c, \min } & \leq V_{B E S S, j, t}^{d c} \leq V_{B E S S, j}^{d c, \max } \\
I_{B E S S, j}^{d c, \min } & \leq I_{B E S S, j, t}^{d c} \leq I_{B E S S, j}^{d c, \max }
\end{aligned}
$$

The constraints of (18) are related to:

\section{a) DC voltage constraints:}

By rewriting (14), we can obtain $P_{B E S S, j, t}^{d c}$ as a function of $V_{B E S S, j, t}^{d c}$ shown by (19). This function is plotted in Fig. 3. It is clear that the part related to the negative DC voltage is not feasible (blue-shaded area).

$$
P_{B E S S, j, t}^{d c}=f^{-1}\left(V_{B E S S, j, t}^{d c}\right)=\left(\frac{1}{D}\right) V_{B E S S, j, t}^{d c}{ }^{2}-\frac{(C X)}{D} V_{B E S S, j, t}^{d c}
$$

Note that when charging, $V_{B E S S, j, t}^{d c}$ increases. Thus, the maximum $P_{B E S S, j, t}^{d c}$ is obtained during charging phase considering the maximum limit of the DC voltage $\left(V_{B E S S, j}^{d c, \max }\right)$. On the contrary, during the discharge, $V_{B E S S, j, t}^{d c}$ decreases and the minimum $P_{B E S S, j, t}^{d c}$ is reached only during discharging phase considering the minimum limit of the DC voltage $\left(V_{B E S S, j}^{d c, \min }\right)$. Moreover, it should be noted that the pink-shaded area in the discharging phase is not feasible due to the continuity reason.

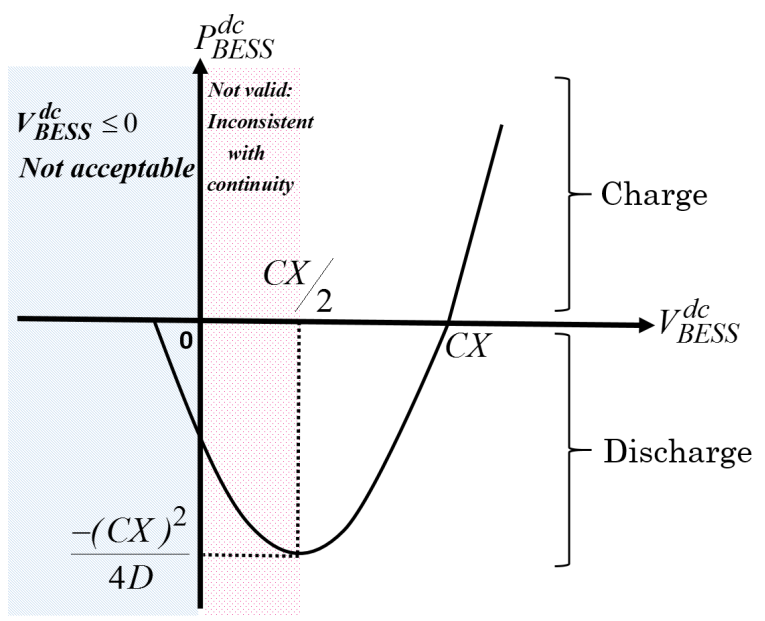

Figure 3. DC power of BESS as a function of DC voltage

The maximum/minimum DC power accounting for the DC voltage constraints are $P_{B E S S}^{d c, \max V}$ and $P_{B E S S}^{d c, \min V}$ respectively. These limits are computed by (20).

$$
\left\{\begin{array}{l}
P_{B E S S, j, t}^{d c, \max V}=f^{-1}\left(V_{B E S S, j}^{d c, \max }\right) \\
P_{B E S S, j, t}^{d c, \min V}=f^{-1}\left(\max \left(V_{B E S S, j}^{d c, \min }, \frac{C X}{2}\right)\right)
\end{array}\right.
$$

\section{b) DC current constraints:}

Eq. (21) is derived from (11) and (14). This function $(g)$ is plotted in Fig. 4. It is seen that the pink-shaded area is not feasible due to the continuity reason. By increasing $I_{B E S S, j, t}^{d c}$, $P_{B E S S, j, t}^{d c}$ increases. Thus, the maximum of $P_{B E S S, j, t}^{d c}$ $\left(P_{B E S S, j, t}^{d c, \max I}\right)$ is related to the $I_{B E S S, j}^{d c, \max }$. However, during discharging phases with negative current flow, the minimum of $P_{B E S S, j, t}^{d c}\left(P_{B E S S, j, t}^{d c, \min I}\right)$ can be found among the ones related to the $I_{B E S S, j}^{d c, m i n}$ and DC power critical point.

$$
\begin{aligned}
& P_{B E S S, j, t}^{d c}=g\left(I_{B E S S, j, t}^{d c}\right)=D I_{B E S S, j, t}^{d c}{ }^{2}+(C X) I_{B E S S, j, t}^{d c} \\
& \left\{\begin{array}{l}
P_{B E S S, j, t}^{d c, \max I}=g\left(I_{B E S S, j}^{d c, \max }\right) \\
P_{B E S S, j, t}^{d c, \min I}=g\left(\max \left(I_{B E S S, j}^{d c, \min }, \frac{-\mathbf{C X}}{2 \mathbf{D}}\right)\right)
\end{array}\right.
\end{aligned}
$$
(23).

As a results a set of dynamic constraints are defined by

$$
\left\{\begin{array}{l}
P_{B E S S, j, t}^{d c, \max }=\min \left(P_{B E S S, j, t}^{d c, \max I}, P_{B E S S, j, t}^{d c, \max V}\right) \\
P_{B E S S, j, t}^{d c, \min }=\max \left(P_{B E S S, j, t}^{d c, \max I}, P_{B E S S, j, t}^{d c, \max V}\right)
\end{array}\right.
$$

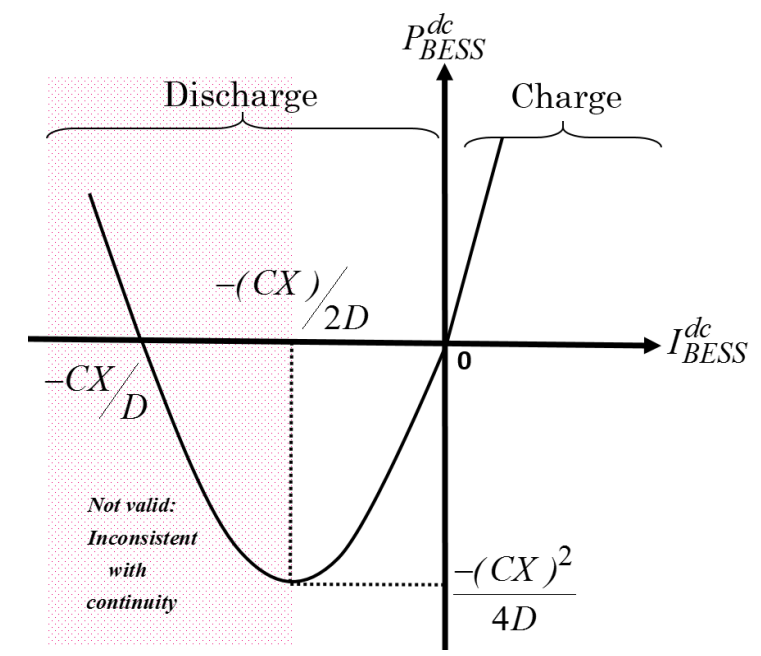

Figure 4. DC power of BESS as a function of DC current

\section{PERFormance EVAlUAtion}

The performance evaluation of the proposed control method is carried out making reference to the 13 nodes IEEE test grid [12]. The network loads and PV injections are inferred from a real feeder of the $20 \mathrm{kV}$ ADN on EPFL campus [13]. Non-dispatchable DG units (PVs) are connected to the system at buses 4,8 and 10 . Their power injections are represented by voltage-independent active power injections 
with null reactive component ${ }^{1}$. The network loads are considered as voltage independent PQ absorptions. The schematic of the system is shown in Fig. 5. The aggregation of loads apparent power absorptions and PVs active power injections for a given day is shown in Fig. 6. The proposed control methods are applied in the system considering three BESSs located at buses 4, 6 and 12 .

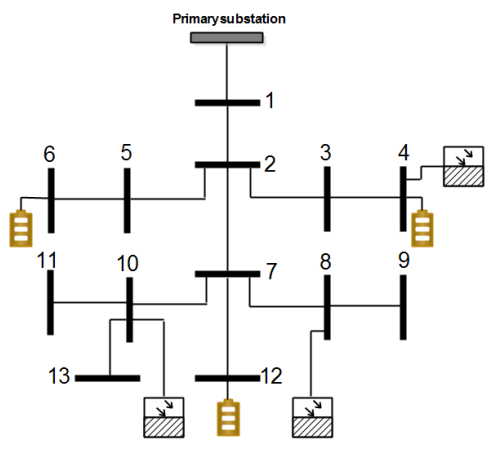

Figure 5. IEEE 13 node test system
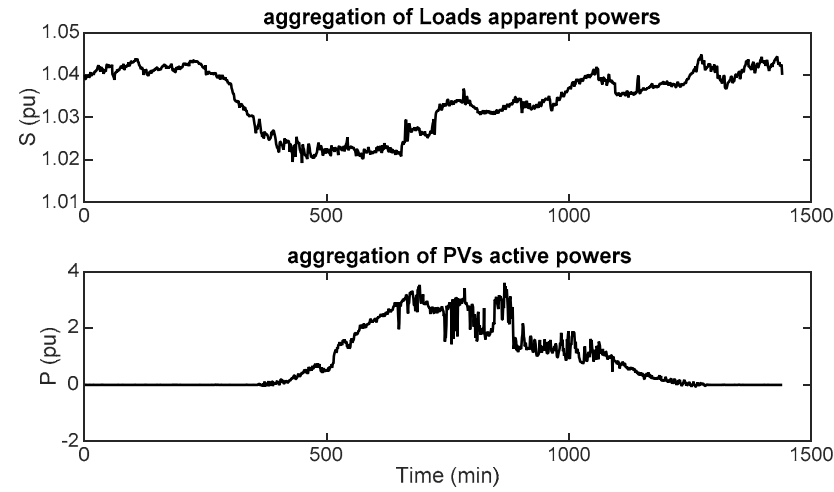

Figure 6. Aggregation of loads/PVs absorptions/injections (base power: $5 \mathrm{MW})$

The optimization problem in (4) is solved using YALMIP optimizer [14]. The two-presented models for BESS in section II are compared in order to see the influence of the accurate BESS model in the grid voltage control. Fig. 7 shows the $S o E$ of the three BESSs for both presented models. It is worth observing that, even if the $S o E$ computation for the two considered BESS model is the same (e.g., from (1)) the set-point computed by the optimal controller are different.

From Fig. 7, it can be seen that a lower energy net flow is requested by the central controller when BESSs are modeled using the TTC model. The DC powers of BESSs for both models are shown in Fig. 8-10. It is evident the action of the computed DC power limits in the TTC model. As it is seen, using the ER model, the DC powers asked from the BESSs

\footnotetext{
${ }^{1}$ It should be noted that reactive power compensation from only PV inverters could be sufficient to keep the acceptable voltage profiles only in network with low $\mathrm{R} / \mathrm{X}$ ratios of longitudinal line impedance parameters, whereas in ADNs normally the feeder $\mathrm{R} / \mathrm{X}$ ratio might be high (see the analysis reported in [11] ). Moreover, the operation of PV inverters with nonunity power factor will result in lower active power production [15]. As a result, in this paper it is considered that the control of the grid is obtained only using BESSs.
}

from the central controller can violate operational DC limits of the BESS.

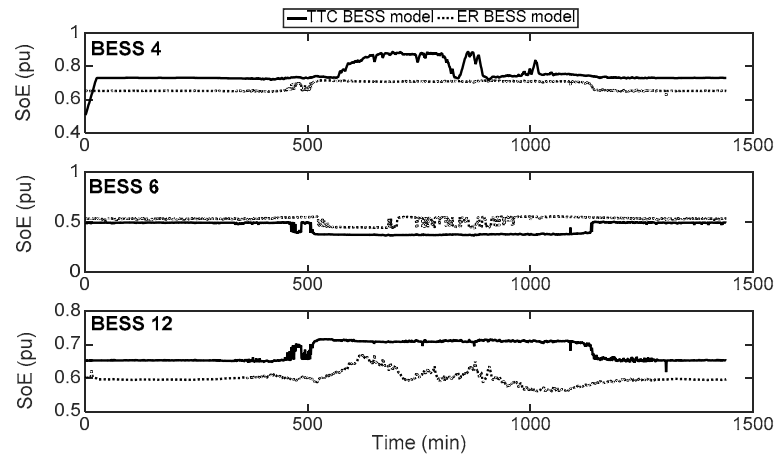

Figure 7. SoE for the two ER and TTC model of BESS

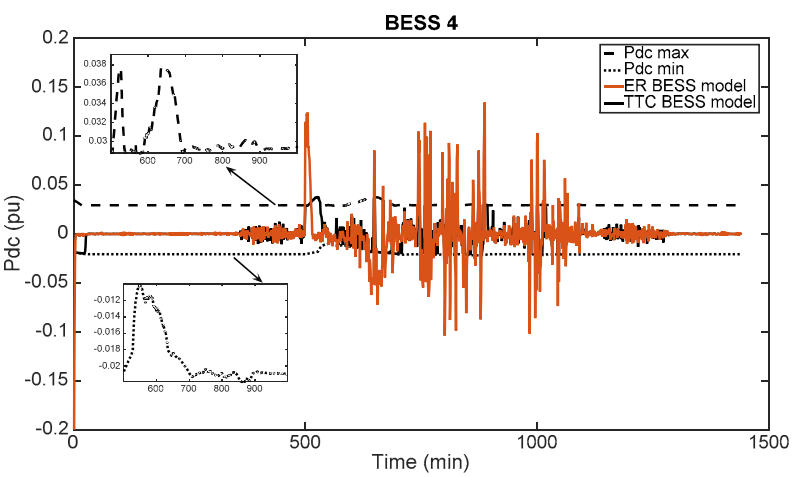

Figure 8. DC power of BESS 4 for the ER and TTC models.

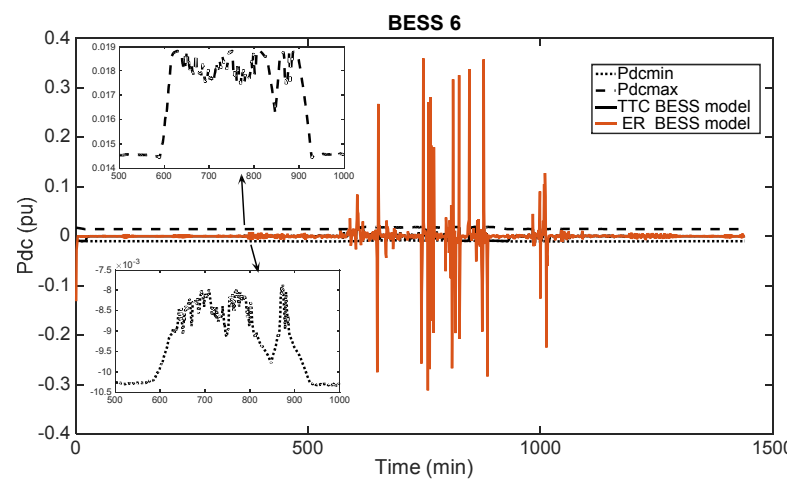

Figure 9. DC power of BESS 6 for the ER and TTC models.

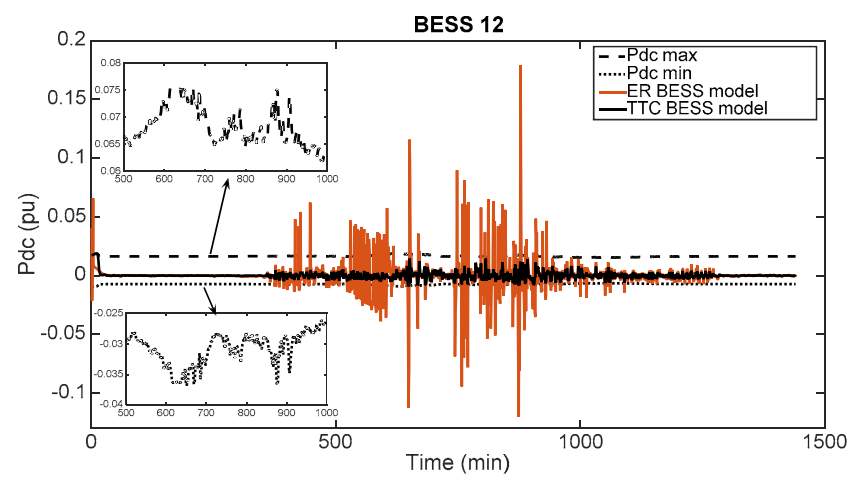

Figure 10. DC power of BESS 12 for the ER and TTC models. 


\section{REFERENCES}

Fig. 11 shows, the maximum, minimum and average values of all the network node voltages for the two cases as well as the case with no BESSs control. As it can be seen, the control coupled with the TTC model leads to slightly worst voltage profiles due to the limiting action of the BESS DC constraints. However, the solution provided by this controller always satisfy the operational limits of the BESSs and, therefore, provide feasible solutions with respect to the constraints of these devices.
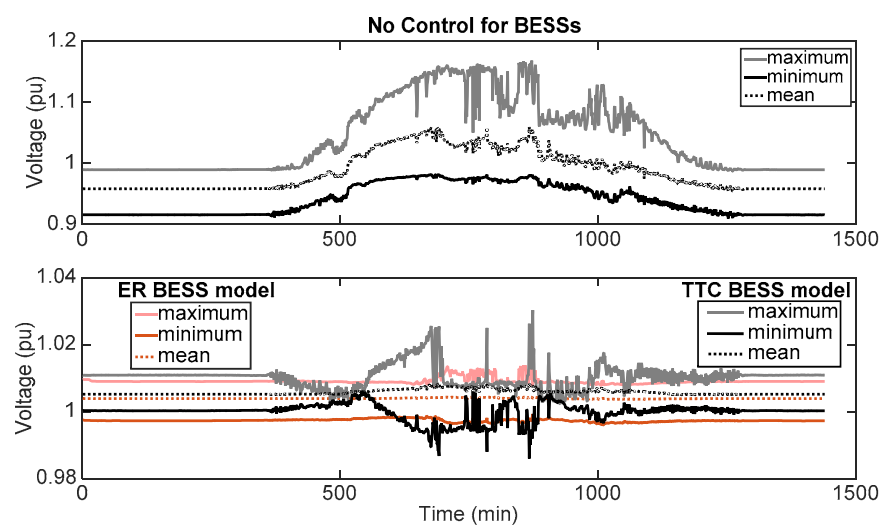

Figure 11. Distribution of all node voltages for the three cases: 1) no control for BESS, 2) ER model of BESS, 3) TTC model of BESS.

\section{CONCLUSIONS}

The paper has proposed an adaptive model-based real-time grid voltage control relying on the accurate dynamic model of the Battery Energy Storage Systems (BESSs). Two different BESSs models are presented, namely an Energy Representative (ER) model and a Two Time Constant (TTC) model. These two models have been considered in a central control algorithm targeting the voltage control of Active Distribution Networks in presence of stochastic resources.

For the case of the TTC model, the paper has discussed the analytical computation of the battery DC constraints to be used in the optimization problem solved by the central controller. The performances of the considered central controller are compared with those obtained for the case where BESSs are modeled as ideal energy reservoirs. Such an assessment is carried out using a numerical example referring to IEEE 13 buses distribution test feeder suitably adapted to include stochastic generation and BESSs.

It is shown that the control coupled with the TTC model leads to slightly worst voltage profiles due to the limiting action of the BESS DC constraints. However, the solution provided by this controller always satisfy the operational limits of the BESSs and, therefore, provide feasible solutions with respect to the constraints of these devices. On the contrary, the adoption of the ER model might provide unfeasible solution since the DC voltage/current limits of BESSs may not be respected.
[1] N. Hatziargyriou, "Microgrids : Architectures and Control," no. WileyIEEE Press, p. 340 pages, 2014.

[2] B. P. Roberts and C. Sandberg, "The Role of Energy Storage in Development of Smart Grids," Proceedings of IEEE, vol. 99, no. 6, pp. 1139-1144, Jun. 2011.

[3] T. Tsuji, K. Tomura, T. Oyama, T. Hashiguchi, T. Goda, S. Tange, and T. Nomura, "A study of centralized voltage profile control of distribution network considering dynamics of distributed generator," Electrical Engineeringin Japan, vol. 179, no. 1, pp. 29-39, Apr. 2012.

[4] M. Bahramipanah, M. Nick, R. Cherkaoui, and M. Paolone, "Network Clustering for Voltage Control in Active Distribution Network Including Energy Storage Systems," in Innovative Smart Grid Technology IEEE PES Conference ISGT, 2015.

[5] M. Nick, R. Cherkaoui, and M. Paolone, "Optimal Allocation of Dispersed Energy Storage Systems in Active Distribution Networks for Energy Balance and Grid Support," IEEE Transaction on Power System, vol. 29, no. 5, pp. 2300-2310, 2014.

[6] B. Belvedere, M. Bianchi, A. Borghetti, C. A. Nucci, M. Paolone, and A. Peretto, "A Microcontroller-Based Power Management System for Standalone Microgrids With Hybrid Power Supply," IEEE Transaction on. Sustainable. ENERGY, vol. 3, no. 3, pp. 422-431, 2012.

[7] A. Rahmoun and H. Biechl, "Modelling of Li-ion batteries using equivalent circuit diagrams," PRZEGLACD ELEKTROTECHNICZNY (Electrical Rev. ISSN 0033-2097, R. 88 NR 7b, vol. 2, no. 7, pp. 152156, 2012.

[8] M. Ecker, S. Kabitz, I. Laresgoiti, and D. U. Sauer, "Parameterization of a Physico-Chemical Model of a Lithium-Ion Battery: II. Model Validation," Journal of Electrochemical Society., vol. 162, no. 9, pp. A1849-A1857, 2015.

[9] M. Ecker, T. K. D. Tran, P. Dechent, S. Kabitz, a. Warnecke, and D. U. Sauer, "Parameterization of a Physico-Chemical Model of a Lithium-Ion Battery: I. Determination of Parameters," Journal of. Electrochem. Soc., vol. 162, no. 9, pp. A1836-A1848, 2015.

[10] M. Bahramipanah, D. Torregrossa, R. Cherkaoui, and M. Paolone, "Enhanced Electrical Model of Lithium-Based Batteries Accounting the Charge Redistribution Effect," in Power System Computation Conference (PSCC), 2014

[11] K. Christakou, J.-Y. Le Boudec, M. Paolone, and D.-C. Tomozei, "Efficient Computation of Sensitivity Coefficients of Node Voltages and Line Currents in Unbalanced Radial Electrical Distribution Networks," IEEE Transaction on Smart Grid, vol. 4, no. 2, pp. 741 - 750, 2012.

[12]"IEEE PES Distribution System Analysis Subcommittee's Distribution Test Feeder Working Group."

[13] M. Pignati, M. Popovic, S. Barreto Andrade, R. Cherkaoui, D. Flores, J.-Y. Le Boudec, M. M. Maaz, M. Paolone, P. Romano, S. Sarri, T. T. Tesfay, D.-C. Tomozei, and L. Zanni, "Real-Time State Estimation of the EPFL-Campus Medium-Voltage Grid by Using PMUs," in Innovative Smart Grid Technologies Conference (ISGT), 2015, pp. 1-5.

[14] J. Efberg, "YALMIP : A toolbox for modeling and optimization in MATLAB," in IEEE International Symposium on Computer Aided Control Systems Design, 2004, pp. 284-289.

[15] M. N. Kabir, Y. Mishra, G. Ledwich, Z. Y. Dong, and K. P. Wong, "Coordinated Control of Grid-Connected Photovoltaic Reactive Power and Battery Energy Storage Systems to Improve the Voltage Pro fi le of a Residential Distribution Feeder," IEEE Transaction on Industrial Informatics, vol. 10, no. 2, pp. 967-977, 2014. 